\title{
Betriebliche Umweltinformationssysteme
}

\author{
Horst Junker', Jorge Marx Gómez², Corinna V. Lang³ \\ ${ }^{1} I M B C G m b H$ \\ 2 Abteilung Wirtschaftsinformatik I, \\ Carl-von-Ossietzky-Universität Oldenburg \\ ${ }^{3}$ Fachbereich Wirtschaft, Hochschule Anbalt
}

\section{Einleitung}

In den Jahren 2006 und 2009 wurden von El-Gayar und Fritz (2006, S. 756-784) sowie von Teuteberg und Straßenburg (2009, S. 64-77) Übersichten über den jeweils aktuellen wissenschaftlichen Entwicklungsstand Betrieblicher Umweltinformationssysteme vorgelegt. So verdienstvoll diese Untersuchungen sind, lassen sie doch den Leser der dort dokumentierten Ergebnisse aus mindestens drei Gründen unbefriedigt zurück.

Zum einen wird deutlich, dass sich die unter dem Rubrum Betriebliche Umweltinformationssysteme publizierten Forschungsergebnisse häufig nur mit Teilaspekten ganzheitlicher Umweltinformationssysteme auseinander setzen und nicht wie beispielsweise die aus der Wirtschaftsinformatik bekannten ERP- oder Business Intelligence-Systeme unternehmensumfassende Funktionalitäten bereitstellen. Zum anderen fällt auf, dass die in diesen Publikationen skizzierten Informationssysteme ganz überwiegend keinen proaktiven Charakter besitzen. Vereinfachend (und ein wenig polemisch) lässt sich konstatieren, dass diese Informationssysteme nicht dahingehend ausgelegt sind, betriebliche Umweltschäden und -belastungen zu reduzieren oder gar zu vermeiden. Vielmehr dienen diese Systeme - wie beispielsweise nahezu alle Abfallmanagementsysteme - dazu, mit bereits vorliegenden Umweltbelastungen (effizient) umzugehen. Zum dritten wird die für eine anwendungsorientierte Wissenschaftsdisziplin sehr wichtige Frage nach dem erfolgreichen Transfer der Forschungsergebnisse in die unternehmerische Praxis kaum gestellt.

Aufgrund des vorliegenden Erfahrungshintergrundes durch mehr als 40 in Unternehmen durchgeführte (Teil-)Projekte (Junker 2004, Junker und Rey 2005, Junker und Lang 2006, Junker und Molloy 2007) und Gespräche mit Vertretern von Unternehmensleitungen von weit mehr als 100 Unternehmen ist festzustellen, dass nur wenige der bislang von der Betrieblichen Umweltinformatik bereitgestellten Forschungsergebnisse Eingang in die betriebliche Praxis gefunden haben. Die 
Durchdringung der Unternehmen mit betrieblichen Umweltinformationssystemen ist gegenwärtig noch nicht in einen erforderlichen und wünschenswerten - und die Ergebnisse der Nachhaltigkeitsdiskussion förderlichen - Umfang gelungen. Die Praxis zeigt, dass insbesondere die Unternehmensleitungen von der Effektivität dieser Systeme kaum überzeugt sind.

Vor dem Hintergrund dieser umfangreichen praktischen Erfahrungen versuchen die nachstehenden Überlegungen - gelegentlich ein wenig zugespitzt - einige Ursachen für diesen Umstand aufzugreifen, um letztlich eine als dringend und zwingend erforderlich angesehene Diskussion mit dem Ziel zu fördern, einen Paradigmenwechsel für die Disziplin der Betrieblichen Umweltinformatik einzufordern.

\section{Traditionelle Betriebliche Umweltinformationssysteme}

Wenn auch die Betriebliche Umweltinformatik im letzten Jahrzehnt einige durchaus anerkennenswerte Erfolge zu verzeichnen hat, beispielsweise in den Bemühungen, Betriebliche Umweltinformationssysteme mit ERP-Systemen zu integrieren, oder durch die Bereitstellung praxisnaher Referenzmodelle und Prototypen, scheinen die Erkenntnisfortschritte dieser Disziplin insbesondere im Vergleich zur Dekade zuvor um Etliches geringfügiger zu sein. Der (teilnehmende) Beobachter der Betrieblichen Umweltinformatik gewinnt den Eindruck, dass inzwischen eine gewisse Ernüchterung eingekehrt ist, die möglicherweise den geringen Erfolg betrieblicher Umweltinformationssysteme im unternehmenspraktischen Einsatz zur Ursache hat.

Grundsätzlich setzen sich Betriebliche Umweltinformationssysteme (BUIS) mit umweltorientierten Phänomenen innerhalb von Unternehmen auseinander. M. a. W. handelt es sich somit um Informationssysteme, die sich mit dem Aufgabenspektrum einer spezifischen betrieblichen Sphäre beschäftigen.

Zunächst sind BUIS aber Informationssysteme, die gemäß Balzert Einheiten aus organisatorischen Systemen zusammen mit sonstigen technischen Einheiten darstellen (Balzert 1996, S. 23). Sie bestehen aus Menschen und Maschinen, die ihre Aufgaben kooperativ ausführen, die mittels Kommunikationsbeziehungen verbunden sind und diese zur Erzeugung, Speicherung, Verarbeitung und Übermittlung von Informationen benutzen. Ein solches Aufgaben-Technik(Organisation)-Mensch-System dient der Erfüllung definierter Aufgaben (Teubner 1999, S. 20).

Im Ergebnis werden unter Informationssystemen solche Systeme verstanden, die durch IT-Systeme unterstützt werden, d. h. maschinelle Aufgabenträger besitzen, und eine konkrete betriebliche Aufgabe oder einen Aufgabenbereich unterstützen. In dieser allgemeinen Definition sind die Eigenschaften und Aufgaben von Informationssystemen auf BUIS übertragbar. 
In der Geschichte der Unternehmen gerieten Umwelt(schutz)aktivitäten erst relativ spät in den Fokus des Interesses der Unternehmensleitungen - etwa zu Ende der 80er Jahre des letzten Jahrhunderts. Die Integration von Umweltaktivitäten in das Aufgabenspektrum von Unternehmen wurde einerseits forciert durch eine beginnende Umweltgesetzgebung, die auch die Unternehmen in die Pflicht nahm. Der Gesetzgeber war zunehmend weniger willens, den Unternehmen die Möglichkeit zu eröffnen, die mit der Produktion von Gütern entstehenden Kosten für die Beseitigung von Umweltschädigungen zu externalisieren. Darüber hinaus wurden mit der ISO 14.001 und der EMAS zwei Normen für Umweltmanagementsysteme publiziert, denen sich die Unternehmen freiwillig unterwerfen konnten. Insgesamt wurde die betriebliche Auseinandersetzung mit Umweltphänomenen weitgehend extern initiiert.

Gegenstand von Umweltmanagementsystemen ist die natürliche Umwelt, letztlich die Natur. Sie „,ist die Gesamtheit aller Faktoren in Form von Luft, Wasser, Boden erneuerbarer und nicht erneuerbarer Rohstoffe und Energie, Wetter, Klima und ökosystemaren Prozesse, die auf das Unternehmen einwirken oder auf die das Unternehmen einwirkt.“ (Müller-Christ 2001, S. 4)

Empirische Studien zeigen allerdings auf, dass sich in der Praxis der Unternehmen die Maßnahmen im Rahmen der Umweltmanagementsysteme auf die operative Ebene konzentrieren und somit eher nach innen gerichtet sind. Betriebliche Optimierungen und die Ausnutzung von Potenzialen zur Kostenreduzierung sind vorherrschend. Für weitergehende marktorientierte Strategien und ökologische Produktinnovationen werden Umweltmanagementsysteme kaum genutzt. Die ökologische Wirksamkeit von Umweltmanagementsystemen wird wesentlich von externen Anreizen aus Politik, Markt und Gesellschaft beeinflusst. Fraglich bleibt, ob der Einsatz von Umweltmanagementsystemen zur Formulierung von anspruchsvollen Umweltzielen führt. (Hamschmidt 2001, S. 59)

Insgesamt muss konstatiert werden, dass das Ziel, Umweltmanagement in den Fokus des allgemeinen (strategischen) Unternehmensmanagement zu integrieren in der Praxis der Betriebe weitgehend misslungen ist Die Einflussnahme der Umweltabteilungen und der -beauftragten auf die strategischen Unternehmensziele ist denkbar gering. Nach wie vor werden die obersten Leitungsebenen der Unternehmen von dem nur schwer erschütterbaren Glauben getragen, dass betriebliche Umweltschutzaktivitäten ausschließlich einen erheblichen Kostenfaktor darstellen.

Damit ist die Planung, Steuerung, Durchführung und Kontrolle von Umweltmaßnahmen weitgehend der operativen Führungsebene zugeordnet. Als Folge der in den letzten drei Jahrzehnten entstandenen Gesetzes- und Vorschriftenflut, die den Unternehmen umfangreiche Beschränkungen und Überwachungspflichten sowie deren Dokumentation und Nachweisführung auferlegten, entstanden dezentrale Anforderungen, die häufig durch dezentrale IT-Lösungen mit sehr unterschiedlichen Erfolgs- und Qualitätsniveaus abgedeckt wurden. Solche Insellösungen erfordern eine wenig komfortable Datenhaltung und verursachen häufig zeitund kostspielige Mehrarbeit. Für viele Unternehmen steht der damit verbundene 
Lern-, Zeit- und Kostenaufwand in keinem akzeptablen Verhältnis zur Anwendungshäufigkeit und dem Nutzen dieser Programme.

Auf der Grundlage dieser Sachverhalte werden bislang in Wissenschaft und Praxis die vielfältigsten Anwendungssysteme als Betriebliche Umweltinformationssysteme bezeichnet, ohne dass sich eine durchgehende und allgemein verbindliche Definition durchgesetzt hat. (Schlatter 2000, S. 2) Meistens wird jeglicher Einsatz von Informationstechnik zur Bearbeitung von umweltrelevanten Daten als BUIS bezeichnet. Nachfolgend werden einige BUIS-Definitionen aus der deutschsprachigen Literatur vorgestellt.

- [Ein BUIS ist] ein betriebwirtschaftliches Instrument für vorausschauendes, langfristiges, strategisches und innovatives Handeln [...], das ökologische Chancen und Risiken aufdecken kann. [...] Ein betriebliches Umweltinformationssystem dient nicht nur der Dokumentation im weitesten Sinne, sondern auch der Planung, Überwachung und Steuerung. (Schulz 1991, S. 225)

- Ein BUIS ist die Zusammenfassung aller Methoden und Instrumente, die auf die Abbildung und Bewertung der von einem Unternehmen ausgehenden ökologischen Wirkungen gerichtet ist. (Zahn und Steimle 1993, o.S.)

- Unter dem Begriff BUIS [werden] alle dem unternehmensweiten Umweltmanagement dienenden Informationssysteme zusammengefasst. (Kramer 1993, S. 10)

- Ein betriebliches Umweltinformationssystem (BUIS) stellt ein Subsystem betrieblicher Informationssysteme zur Unterstützung des Umweltmanagements dar. (Eschenbach und Neumann 1995, S. 76)

- Betriebliche Umweltinformationssysteme (BUIS) dienen der informationstechnischen Unterstützung des betrieblichen Umweltschutzes. (Hilty 1997, S. 84)

- Ein betriebliches Umweltinformationssystem (BUIS) ist ein organisatorischtechnisches System zur systematischen Erfassung, Verarbeitung und Bereitstellung umweltrelevanter Informationen in einem Betrieb. (Rautenstrauch 1999, S. 11)

Alle vorgenannten Definitionen vermitteln den Eindruck eines hohen Maßes an Unschärfe. Dieser Sachverhalt ist offenbar der Tatsache geschuldet, dass die Autoren (auch) den Versuch unternommen haben, die Charakteristika real existierender umweltorientierter Softwaresysteme in ihren Definitionen „einfangen“ zu wollen. Diese Vermutung wird durch den nachfolgenden Definitionsversuch bestätigt: „In Analogie zu betrieblichen Informationssystemen ist ein Betriebliches Umweltinformationssystem ein Informationssystem, das für die Erfassung, Dokumentation, Planung und Steuerung betrieblicher Umweltwirkungen genutzt wird und das betriebliche Umweltmanagement in seinen Aufgaben unterstützt. Unter Informationssysteme werden zudem Systeme unterschiedlicher Komplexität verstanden. Ein BUIS kann demnach sowohl eine Tabellenkalkulation zur Berechnung von Um- 
weltkennzahlen als auch eine spezifische Software zur Analyse von Stoffströmen oder zur Bilanzierung von Umweltwirkungen im Rahmen der Ökobilanzierung sein.“ (Beucker 2002, S. 9)

In der Tat lassen sich diese Ausprägungen unter die oben genannten Definitionen subsumieren. Allerdings wird damit der Definitionsrahmen für Informationssysteme der Wirtschaftinformatik weit überschritten. Dort genannte Eigenschaften von Informationssystemen, die grundsätzlich auch auf BUIS zutreffen müssen, sind u. a.:

- Einheitlichkeit der Benutzerschnittstelle, der Daten- und Prozessmodelle usw.,

- Integration vorhandener Teillösungen mit inhaltlichen Berührungspunkten,

- unternehmensweiter Einsatz,

- medienübergreifenden Einsatz,

- Wirtschaftlichkeit,

- Benutzerfreundlichkeit,

- Sicherung von Datenschutz, -integrität und -sicherheit,

- Einhaltung von Standards.

Die große Mehrheit dieser Kriterien findet in den vorliegenden Definitionen von BUIS nicht oder in einem nur sehr geringem Maße Widerhall. Genau diese mangelhafte Berücksichtigung der zuvor genannten Kriterien kann dann dazu führen, dass die Anwendung eines eher trivialen Tabellenkalkulationsprogramms als ein Betriebliches Umweltinformationssystem interpretiert werden kann - und interpretiert wird.

Die Unschärfe der BUIS-Definitionen sowie die Vielfalt an in den Unternehmen befindlichen umweltorientierten Anwendungssystemen hat dazu geführt, einen morphologischen Kasten als Klassifikationsschema für BUIS zu verwenden. (Rey und Schnapernelle 1999, S. 149)

Für den praktischen Einsatz von BUIS in den Unternehmen führten die dargestellten Definitionen dazu, dass faktisch jede IT-gestützte umweltorientierte Problemlösung ein BUIS darstellt, mag der damit gelöste Problemumfang noch so gering und noch so wenig komplex sein. Damit führen die - traditionellen - Definitionen des Begriffs BUIS wegen ihrer stark operativen Ausrichtung zur Weigerung der Unternehmensleitungen, die Aufgaben des Umweltmanagement und deren IT-seitige Unterstützung in den Kranz ihrer strategischen Managementaufgaben aufzunehmen.

Es ist auffällig, dass keiner der Autoren, die sich um BUIS-Definitionen bemüht haben, das Ideengerüst der spätestens seit dem Weltgipfel 1992 in Rio de Janeiro vielfältig und breit geführten Nachhaltigkeitsdebatte aufgegriffen haben, sondern sich vielmehr an die vergleichsweise enge Begrifflichkeit der Umwelt orientiert haben. Aus diesem Grunde ist es wenig verwunderlich, dass die Planer und Entscheidungsträger in den Unternehmen bei der Realisierung ihrer BUIS sich 
eher dem Gedanken des sog. passiven Umweltschutz verpflichtet sahen, bei dem es im Wesentlichen darauf ankommt, den sich aus der Forderung nach Gesetzes

\begin{tabular}{|c|c|c|c|c|c|c|c|c|c|c|c|c|}
\hline & Merkmal & & & & & & Ausprä & ägung & & & & \\
\hline \multirow{5}{*}{ 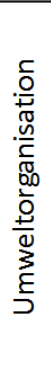 } & Strategie & \multicolumn{6}{|c|}{ Präventiv } & \multicolumn{5}{|c|}{ Nachsorgend } \\
\hline & $\begin{array}{l}\text { Unternehmens- } \\
\text { ziel }\end{array}$ & \multicolumn{2}{|c|}{$\begin{array}{c}\text { EMAS/ISO- } \\
\text { Zertifizierung }\end{array}$} & \multicolumn{4}{|c|}{$\begin{array}{c}\text { Umweltoptimierung/ } \\
\text { Ökoeffizienz }\end{array}$} & \multicolumn{3}{|c|}{$\begin{array}{c}\text { Erfüllen gesetzlicher } \\
\text { Umweltauflagen }\end{array}$} & \multicolumn{2}{|c|}{$\begin{array}{l}\text { Darstellung der } \\
\text { Umweltleistung }\end{array}$} \\
\hline & Zeithorizont & \multicolumn{4}{|c|}{ Strategisch langfristig } & \multicolumn{4}{|c|}{ Taktisch mittelfristig } & \multicolumn{3}{|c|}{ Operativ kurzfristig } \\
\hline & $\begin{array}{l}\text { Organisations- } \\
\text { einheit }\end{array}$ & \multicolumn{2}{|c|}{ Management } & \multicolumn{4}{|c|}{ Fachbereich } & \multicolumn{3}{|c|}{ Umweltbeauftragter } & \multicolumn{2}{|c|}{$\begin{array}{c}\text { Dezentrale } \\
\text { Verantwortung }\end{array}$} \\
\hline & Einsatzbereich & $\begin{array}{c}\text { Abfallwirt- } \\
\text { schaft }\end{array}$ & $\begin{array}{c}\text { Gewässer- } \\
\text { schutz }\end{array}$ & & $\begin{array}{l}\text { mis- } \\
\text { ions- } \\
\text { chutz }\end{array}$ & $\begin{array}{r}\text { Ene } \\
\text { maß } \\
\mathrm{m}\end{array}$ & $\begin{array}{l}\text { rgie- } \\
\text { Snah- } \\
\text { ien }\end{array}$ & $\begin{array}{r}\text { Gefahrs } \\
\text { mana } \\
\text { men }\end{array}$ & $\begin{array}{l}\text { stoff- } \\
\text { ge- } \\
\text { te }\end{array}$ & \begin{tabular}{|c|} 
Stoff- \\
datenver- \\
waltung
\end{tabular} & $\begin{array}{c}\text { Anlagen- } \\
\text { verwal- } \\
\text { tung }\end{array}$ & $\begin{array}{c}\text { Stoffstrom- } \\
\text { manage- } \\
\text { ment }\end{array}$ \\
\hline \multirow{3}{*}{ 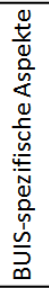 } & Aufgaben & \begin{tabular}{|c|} 
Bericht- \\
erstellung
\end{tabular} & \begin{tabular}{|c|} 
Unterstütz- \\
ung der \\
Prozess- \\
planung
\end{tabular} & $\begin{array}{l}\text { Unte } \\
\text { un } \\
\text { Pro } \\
\text { steu }\end{array}$ & $\begin{array}{l}\text { erstütz- } \\
\text { ig der } \\
\text { ozess- } \\
\text { uerung }\end{array}$ & $\begin{array}{r}\text { Unte } \\
\text { ung } \\
\text { Proz } \\
\text { berwa }\end{array}$ & $\begin{array}{l}\text { rstütz- } \\
\text { gder } \\
\text { zessü- } \\
\text { achung }\end{array}$ & $\begin{array}{r}\text { Vorgeh } \\
\text { unterst } \\
\text { ung/L } \\
\text { fade }\end{array}$ & $\begin{array}{l}\text { nens- } \\
\text { tütz- } \\
\text { eit- } \\
\text { en }\end{array}$ & $\begin{array}{c}\text { Informa- } \\
\text { tions- } \\
\text { schnitt- } \\
\text { stelle }\end{array}$ & $\begin{array}{l}\text { Organisa- } \\
\text { tionsunter- } \\
\text { stützung }\end{array}$ & $\begin{array}{c}\text { Umwelt- } \\
\text { bilan- } \\
\text { zierung }\end{array}$ \\
\hline & Funktionalität & Analyse & $\begin{array}{c}\text { Modellier } \\
\text { ung }\end{array}$ & & Simulat & tion & $\begin{array}{l}\text { Zeitna } \\
\text { Monitc }\end{array}$ & $\begin{array}{l}\text { lahes } \\
\text { toring }\end{array}$ & $\begin{array}{r}\text { Doku } \\
\text { ma } \\
m\end{array}$ & $\begin{array}{l}\text { ument- } \\
\text { anage- } \\
\text { nent }\end{array}$ & $\begin{array}{l}\text { Report- } \\
\text { enerator }\end{array}$ & $\begin{array}{c}\text { Workflow- } \\
\text { Kompo- } \\
\text { nente }\end{array}$ \\
\hline & Systemgrenze & \multicolumn{4}{|c|}{ Bereich/Unternehmen } & \multicolumn{4}{|c|}{ Prozess } & \multicolumn{3}{|c|}{ Produkt/LCA } \\
\hline \multirow{3}{*}{ ヒ } & $\begin{array}{l}\text { Integrations- } \\
\text { grad }\end{array}$ & \multicolumn{3}{|c|}{ Stand-alone } & \multicolumn{5}{|c|}{ Add-On } & \multicolumn{3}{|c|}{ Integriertes System } \\
\hline & Betriebssystem & \multicolumn{2}{|c|}{ Windows } & \multicolumn{4}{|c|}{ Unix } & \multicolumn{3}{|c|}{$\mathrm{OS} / 2$} & \multicolumn{2}{|c|}{ Sonstige } \\
\hline & Formate & \multicolumn{2}{|c|}{ HTML } & \multicolumn{4}{|c|}{ ODBC } & \multicolumn{3}{|c|}{ Office-Formate } & \multicolumn{2}{|c|}{ Sonstige } \\
\hline
\end{tabular}

\section{Abbildung 1: Morphologischer Kasten für BUIS}

konformität ergebenden Sachzwängen durch Vermeidung, mindestens aber durch Reduzierung von Umweltschädigungen nachzukommen. Dabei wurden BUIS primär unter dem Aspekt der Outputorientierung entwickelt. Im Ergebnis wurden durch den Einsatz sog. end of pipe-Technologien Emissionen reduziert oder vermieden, ohne Änderungen an den bestehenden Produktionsprozessen vorzunehmen. Dadurch muss zur Erfüllung der (gesetzlichen) Umweltauflagen ein zusätzlicher Aufwand getrieben werden, der naturgemäß Kosten verursacht. Somit sind wohl insbesondere die passiven Umweltschutzaktivitäten der Unternehmen der wesentliche Ursprung der Managementthese, dass der betriebliche Umweltschutz (nahezu) ausschließlich einen Kostenfaktor darstellt.

\section{Nachhaltige Entwicklung}

Geprägt und bekannt wurde der Begriff der Nachhaltigkeit erst ab 1987 durch den Zukunftsbericht „Our Common Future“, besser bekannt unter der Bezeichnung „Brundlandt-Bericht“. Es war der Abschlussbericht der Weltkommission für Umwelt und Entwicklung (WECD). Dieser Bericht war der wesentliche auslösende 
Faktor für die Konferenz der Vereinten Nationen für Umwelt und Entwicklung1992 in Rio de Janeiro (auch als Weltgipfel bezeichnet). Die Konferenz erklärte eine nachhaltige Entwicklung zur zentralen Zukunftsaufgabe der Gesellschaften für das 21. Jahrhundert. Aus dieser Konferenz ging auch das internationale Aktionsprogramm der Agenda 21 hervor, bei dem sich die Staten, die die Agenda unterzeichneten, verpflichten, den Übergang zu einer nachhaltigen Entwicklung zu fördern. (Rösch und Grossmann 1998, S. 27)

Die langfristige Sicherung der natürlichen Lebensgrundlagen und die Verbesserung der ökonomischen und sozialen Lebensbedingungen bilden die drei Dimensionen, die das Leitbild der nachhaltigen Entwicklung zu vereinbaren sucht. Somit geht dieses Leitbild weit über die Betrachtung der umweltpolitischen Komponente hinaus und berührt unmittelbar ökonomische, ökologische und soziale Entwicklungsprozesse. (Deutscher Bundestag 1997, S. 11)

Als nachhaltig gilt eine Entwicklung dann, wenn sie „die Bedürfnisse der Gegenwart befriedigt, ohne zu riskieren, dass künftige Generationen ihre eigenen Bedürfnisse nicht befriedigen können." (Hauff 1987, S. 46) Wurden zuvor die drei Dimensionen unabhängig voneinander betrachtet, entstand durch das in Bezug setzen der drei Dimensionen eine komplexe Problemdefinition, die es unmöglich macht, einen einheitlichen Lösungsvorschlag zu erhalten. Die drei Dimensionen bedingen sich gegenseitig, jede übt Einfluss auf die anderen aus.

Während der Begriff des Umweltschutzes andererseits durchaus bekannt ist, scheint der Begriff der nachhaltigen Entwicklung keine direkte Botschaft zu vermitteln. Häufig fällt es schwer, sich darunter konkret etwas vorzustellen. (MüllerChrist 2001, S. 523f) Das „Drei-Säulen-Modell“ der Enquetekommission des deutschen Bundestages, das die erwähnten drei Dimensionen reflektiert, stellt einen prozessorientierten Ansatz dar. Dieser setzt nicht voraus, dass das Leitbild einer nachhaltigen Entwicklung mittels Zielen eindeutig bestimmbar ist und als Folge davon entsprechende nachhaltige Handlungsprogramme entworfen werden können. (Heß 2007, S. 10)

Das Ziel einer zukünftigen wirtschaftlich nachhaltigen Entwicklung ist die Realisierung eines Ressourcen sparenden technischen Fortschritts. Die Rohstoffentnahme aus der natürlichen Umwelt sowie die Aufnahmefähigkeit der natürlichen Umwelt sind begrenzt. Die Einwegwirtschaft muss gemäß des Leitbildes der nachhaltigen Entwicklung einer Kreislaufwirtschaft weichen. (Rautenstrauch 1999, S. 2)

Insbesondere durch die Realisierung einer Kreislaufwirtschaft (VermeidenVerwerten-Beseitigen) und eines integrierten Umweltschutzes (Prozess-, Produktions- und Produktintegration) sind erste Möglichkeiten zur Ausgestaltung eines nachhaltigen Wirtschaftens in den Unternehmen gegeben. Um optimale nachhaltigkeitsbezogenen Entscheidungen treffen zu können, sind wirtschaftlich-, umweltund arbeitsplatzrelevante (die drei Leitdimensionen einer nachhaltigen Entwicklung) Informationen als auch Informationen über Rohstoffe, Anlagen, Standort, Nachfrage hinsichtlich von Produkten u. a. bereitzustellen. (Haasis 2001 S. 27ff). 
Dies führt zu einer nachhaltigen Unternehmensführung, was bedeutet, vermehrt auf interdisziplinäre Information und Wissen zurückzugreifen.

Unter Nachhaltigkeitsaspekten wird das moderne Unternehmen als pluralistische Wertschöpfungseinheit verstanden, die die Forderungen und Erwartungen unterschiedlicher Gruppen zu erfüllen hat, um seine langfristige Existenz zu gewährleisten. Das Anspruchsgruppenkonzept unterstützt die Annahme, dass das langfristige Weiterbestehen eines Unternehmens nur dann sicher gestellt wird, wenn seine wirtschaftlichen Aktivitäten im Einklang mit der Unternehmensumwelt stehen.

Die Ansprüche der verschiedenen Gruppen können sehr heterogen sein. In dieser Heterogenität der Gruppen kann ein Grund für Interessenskonflikte liegen. Dabei hängt die Durchsetzung einzelner Interessen den Unternehmen gegenüber von den Machtverhältnissen und den Rahmenbedingungen zwischen den Interaktionspartnern ab. (Goetz und Czymmek 2002, S. 13f)

Grundsätzlich ist für das Management der Unternehmen der Umgang mit gesellschaftlichen Veränderungsprozessen kein Neuland. Allerdings sehen sich die Unternehmen im Kontext der Nachhaltigkeitsdebatte damit konfrontiert, dass ökologische Ansprüche nicht mehr - wie in der Vergangenheit - allein politisch diskutiert und anschließend als gesetzliche Regelungen oder Gebote und Auflagen umgesetzt werden. Der Staat setzte dadurch eindeutige und klar definierte Rahmenbedingungen. Aus Sicht der Unternehmen gestaltete sich die Umsetzung dieser Ansprüche als externer Prozess. Sie mussten nicht aktiv agieren, sondern lediglich auf gesetzte Rahmenbedingungen reagieren. Heutzutage sind Unternehmen gefordert, aus eigener Motivation ökologische Ansprüche umzusetzen. Sie sind in der Verpflichtung, aktiv zu agieren.

Aus der Nachhaltigkeitsdebatte, die (u. a.) eine Harmonisierung ökonomischer und ökologischer Aspekte fordert, leitet sich somit die Idee eines produktionsintegrierten Umweltschutzes ab, eines sog. aktiven Umweltschutzes, der sich jenseits der Erfüllung gesetzlich vorgegebener Umweltnormen verpflichtet sieht, neben der Vermeidung bzw. Reduktion unerwünschter Emissionen und Abfällen die Material- und Energieeffizienz der Beschaffungs- und Produktionsprozesse zu fördern, um so auch den Forderungen der Anspruchsgruppen Rechnung zu tragen. Für die Entwicklung moderner BUIS ist damit ein ganzheitlicher Ansatz gefordert, der die gesamte Prozesskette (Input-Prozess-Output) unterstützt.

\section{Entwicklung umweltrelevanter betrieblicher Zielsysteme}

Eine unternehmensintern isolierte Betrachtung und Verfolgung umweltrelevanter Ziele hat in der Praxis dazu geführt, dass die Durchführung von Umweltschutzaktivitäten weitgehend der operativen Managementebene zugewiesen wurde, während die ökonomischen Ziele im Interessenfokus des strategischen Managements verblieben. Das führt häufig zu dem Ergebnis, dass der Umweltschutzgedanke Teil des Unternehmensleitbildes sind, faktisch aber nicht gelebt wird. Die im Nachhal- 
tigkeitsbegriff formulierten ökonomische und ökologische Dimensionen machen es erforderlich, dass neben den ökonomischen Kategorien auch der betriebliche Umweltschutz, die Beachtung der schädlichen Auswirkungen, die bei der Produktion von Gütern und Dienstleistungen entstehen, in den Fokus des Managementhandelns zu rücken haben.

Die Erkenntnis, dass umweltrelevante Maßnahmen auch zu positiven ökonomischen Effekten führen können, führt wiederum dazu, dass insbesondere in jüngerer Zeit der betriebliche Umweltschutz mit seinen Zielen der Emissions- und Abfallreduzierung bzw. der Material- und Energieeffizienz zunehmend in das Zielsystem der Unternehmen integriert wird. (Lang 2007a, S. 13f) Umweltrelevante Gesetze und Auflagen geben bereits Eckziele vor und können durch unternehmensinterne Ziele des produktionsintegrierten Umweltschutzes erweitert werden.

Das Aufstellen konkreter Umweltziele, welche sich an einer ökologisch dauerhaften Wirtschaftentwicklung orientieren, verschafft den Unternehmen strategische Klarheit. Insbesondere gegenüber den Anspruchsgruppen ist es erforderlich, klare und nachprüfbare Ziele zu formulieren. Es ist nicht ausreichend, allgemeine Umweltleitlinien und Grundsätze zu formulieren. „Ökologische Ziele müssen konkretisiert und möglichst quantifiziert werden, um sie nachprüfbar zu machen und damit auch Kriterien für Erfolg und Misserfolg zu schaffen." (Bundesumweltamt 2001, S. 4)

Grundsätzlich sind bei Entwicklung von Zielsystemen wichtige Zielkonflikte zu lösen. Untergeordnete Ziele sind beispielsweise sparsame und effiziente Rohstoffbewirtschaftung, die Minimierung von Verpackungen und Transportvorgängen, Stoffunbedenklichkeiten, Reduzierung von Nebenprodukten, Langlebigkeit von Produkten, eine problemlose Entsorgung etc. Es wird deutlich, dass die Umweltziele zur Erreichung des betrieblichen Umweltschutzes der einzelnen Unternehmen je nach Unternehmenstätigkeit sehr unterschiedlich sein können. Nur in abstrakter Form lassen sich alle Unternehmen gleich definieren: „Die Belastung der natürlichen Umwelt muss vermieden werden, indem Emissionen reduziert, Ressourcen geschont und Risiken minimiert werden.“ (Müller-Christ, S. 12)

Es wurde bereits darauf hingewiesen, dass die drei Dimensionen des Nachhaltigkeitsbegriffs geeignet (und notwendig) sind, Unternehmensleitbilder zu generieren und zu etablieren. Allerdings sind Leitbilder nicht hinreichend konkret, um aus ihnen unmittelbar Handlungsanweisungen abzuleiten. Somit besteht eine wichtige Aufgabe darin, Unternehmensziele und deren Konkretisierungen unter Berücksichtigung der unterschiedlichen Interessgruppen zu formulieren, zumal Ziele zentrale Leitgrößen darstellen, an denen zukünftiges Handeln gemessen werden kann. Ziele beschreiben die in der Realität angestrebten Zustände, die durch bestimmte Maßnahmen oder Lösungen erreicht werden sollen.

Sollen Zielsysteme als betriebliches Planungs-, Steuerungs- und Kontrollinstrument dienen, ist es erforderlich, dass die Ziele strukturiert, geordnet, vollständig, allen Beteiligten in den Unternehmen bekannt und transparent sind sowie 
keine Leerstellen, Widersprüche und Unklarheiten bestehen. (Hopfenbeck 1992, S. 502) Somit bedarf es einer systematischen Entwicklung solcher Zielordnungen. Zur Erarbeitung eines so geforderten Zielsystems haben sich in der Praxis die folgenden Arbeitsschritte als zweckmäßig erwiesen:

- Erstellung Zielkatalog

- Aufbau Zielsystem

- Operationalisierung Zielsystem

- Gewichtung Zielsystem

Bei der Entwicklung eines nachhaltigkeitsorientierten Zielsystems ist es notwendig, zunächst für die ökologische und die ökonomische Dimension jeweils getrennt die Oberziele zu definieren. Die anschließende „Bildung von Teil- und Unterzielen hat [...] die Aufgabe, aus dem formalen Oberziel [...] für nachgeordnete Instanzen eine brauchbare Arbeitsanweisung abzuleiten." (Wöhe 2005, S. 101) Die Oberziele werden so mithilfe von Unterzielen zunehmend operationalisiert.

Für nachhaltigkeitsorientierte Systeme sind neben den sich aus der Betriebswirtschaftslehre ergebenden Zielvorstellungen zusätzlich diejenigen zu integrieren, die sich aus dem betrieblichen Umweltmanagement ergeben. Somit ist das Zielsystem eines BUIS - abweichend von den Betrieblichen Informationssystemen und den Umweltinformationssystemen - durch die Integration ökonomischer und ökologischer Teilziele geprägt. (Hilty und Rautenstrauch 1995, S. 296) Die ohnehin vorhandene Komplexität betrieblicher Systeme nimmt durch die zusätzlichen Anforderungen und umweltorientierten Erweiterungen weiter zu und wird zunehmend schwieriger zu beherrschen. „Die Definition der Systemgrenzen hat entscheidenden Einfluss auf die Optimierungspotentiale, die mit dem Systemmodell erschlossen werden können. Je weiter die Grenzen gezogen werden, d. h. je größer der betrachtete Realitätsausschnitt ist, desto weitreichender sind [...] die Möglichkeiten zur Systemoptimierung nach betriebswirtschaftlichen und ökologischen Kriterien.“ (Hilty und Rautenstrauch 1995, S. 301)

Im Folgenden wird versucht, den Nachweis zu führen, dass es möglich ist, ein nachhaltigkeitsorientiertes Zielsystem zu konstruieren. Aus Gründen der Übersichtlichkeit und der Komplexitätsreduzierung werden die ökonomische und die ökologische Dimension der Nachhaltigkeit allerdings nicht auf ein gesamtes Unternehmen bezogen, sondern als Ausschnitt die innerbetriebliche Logistik eines Unternehmens gewählt. Dabei wird im Wesentlichen auf die Arbeitsergebnisse von C. Lang zurückgegriffen. (Lang 2007b)

Auch die innerbetriebliche Logistik als Teilgebiet des gesamten unternehmerischen Handelns leitet ihre Ziele aus den übergeordneten Unternehmenszielen ab. Deshalb verfolgt auch sie kundenorientierte, wettbewerbsentscheidende Ziele, die die Aktivitäten der strategischen, taktischen und operativen Entscheidungsebene bestimmen. (Heiserich 2002, S. 16f) 


\begin{tabular}{|c|c|c|}
\hline Strategische Ebene & Taktische Ebene & Operative Ebene \\
\hline $\begin{array}{l}\text { - Kundengerechte Gestaltung } \\
\text { der Marktversorgung } \\
\text { - Kurzfristige Reaktion auf } \\
\text { Kundenwünsche } \\
\text { - Einstellung auf wechselnde } \\
\text { Marktverhältnisse } \\
\text { - Minimale Logistikkosten } \\
\text { - Erschließung und Absicherung } \\
\text { von } \\
\text { Beschaffungsquellenverbesseru } \\
\text { ng der Materialflüsse mit den } \\
\text { dazugehörigen } \\
\text { Informationsflüssen und } \\
\text { Steuerungssystemen } \\
\text { - Vermeidung der Abhängigkeit } \\
\text { von Lieferanten } \\
\text { - Logistikgerechte } \\
\text { Layoutplanung } \\
\text { - Entscheidung über make or } \\
\text { buy der Entsorgung }\end{array}$ & $\begin{array}{l}\text { - Minimale } \\
\text { Kapitalbindung } \\
\text { - Hohe Produktivität } \\
\text { - Hohe Effizienz } \\
\text { - Hohe Termintreue } \\
\text { - Reduktion des } \\
\text { Versorgungsrisikos } \\
\text { - Verbesserung der } \\
\text { Prozesskette } \\
\text { - Reduzienung von } \\
\text { Versorgungs- und } \\
\text { Bereitstellungskosten } \\
\text { - Sicherung der Qualität } \\
\text { der } \\
\text { Beschaffungsmaterialien } \\
\text { - Minimierung der } \\
\text { Entsorgungskosten }\end{array}$ & $\begin{array}{l}\text { - Hohe und gleichmäßige } \\
\text { Kapazitätsauslastung } \\
\text { - Minimale Bestände } \\
\text { - Kurze Durchlaufzeiten } \\
\text { - Minimale } \\
\text { Terminabweichungen } \\
\text { - Niedrige Einstandspreise } \\
\text { - Hohe Qualität der } \\
\text { Baugruppen } \\
\text { - Rechtzeitiger Abruf der } \\
\text { erforderlichen Mengen } \\
\text { - Kurze Kommissionierzeiten } \\
\text { - Schnelle Angebotserstellung } \\
\text { - Minimaler } \\
\text { Verpackungsmitteleinsatz } \\
\text { - Hohe Recyclingquoten }\end{array}$ \\
\hline
\end{tabular}

Abbildung 2: Zielkatalog für die innerbetriebliche Logistik

Die vorstehende Tabelle gibt die aus der einschlägigen Literatur zusammengestellten ökonomischen Einzelziele des Subsystems Beschaffungs- Produktions-, Vertriebs- und Entsorgungslogistik wieder. (Lang 2007b, S. 44)

Das strategische Umweltmanagement gibt ökologische Unternehmensziele und -strategien vor, die durch die untergeordneten Ebenen weiter zu operationalisieren sind. So lassen sich für eine unternehmensspezifische Umweltpolitik beispielsweise folgende Ziele benennen (vgl. DIN EN ISO 14.001, Nr. 4.2 und Nr.1.4):

- Anerkennung der gesellschaftlichen und umweltpolitischen Verantwortung des Unternehmens,

- Bekenntnis zu den Prinzipien eines vorsorgenden Umweltschutzes,

- Verpflichtung zur Integration der Ziele der nachhaltigen Entwicklung in die betrieblichen Entscheidungsprozesse,

- Berücksichtigung der Tragfähigkeit der Ökosysteme bei der Ressourcennutzung,

- Entwicklung umweltfreundlicher Produktions- und Entsorgungsverfahren sowie

- Bereitschaft zum Dialog und Informationsaustausch mit verschiedenen Anspruchsgruppen.

Daraus abgeleitete Zwischen- und Teilziele zur Umsetzung des betrieblichen Umweltschutzes sind je nach Unternehmenstyp zwar unterschiedlich, jedoch lassen sich die folgenden drei Zielkategorien erkennen (Müller-Christ 2001, S. 16): 
- ressourcenschonende Ziele,

- emissionsbezogene Ziele zur Sicherung der Luft-, Wasser- und Bodenqualität,

- Ziele zur Begrenzung und Vermeidung finanzieller Risiken umweltbezogenen Ursprungs.

Die noch relativ allgemeingültig gehaltenen Ziele der Ressourcenschonung und der Reduzierung von Umweltbelastungen lassen sich als Zwischenziele einordnen, die beispielsweise von der taktischen Ebene des Umweltmanagements definiert werden. So kann das Ziel einer Ressourcenschonung durch die Reduktion von Material- und Energieeinsatzmengen oder auch durch eine Substitution umweltbelastender Materialien und Energiearten umgesetzt werden. Reduzierungen von Umweltbelastungen sind weiterhin durch die Einflussnahme auf die unerwünschten Outputmengen, die infolge der Produktion und der Konsumtion entstehen, möglich. Wenn Abfälle entstehen, ist zu prüfen, inwiefern und in welchen Mengen diese wieder oder weiter zu verwenden oder zu verwerten sind.

Bislang wurde die innerbetriebliche Logistik getrennt hinsichtlich ihrer ökonomischen und ökologischen Zieldimension betrachtet. Ein kombiniertes ökonomisch-ökologisches Zielsystem der innerbetrieblichen Logistik ist auch in das Gesamtzielsystem eines Unternehmens einzubinden. (Wöhe 2005, S. 457; Rautenstrauch 1997, S. 12ff)

Die Steigerung der Wirtschaftlichkeit der Logistik ist ein wichtiges ökonomisches Unternehmensziel. Die innerbetriebliche Logistik hat aber auch für das betriebliche Umweltmanagement einen besonderen Stellenwert, weil hier Materialund Energiekreisläufe sowie Transport, Umschlag- und Lagerprozesse betrachtet werden. Hier getroffene Entscheidungen wirken sich unmittelbar auf die Einsatzmengen der Ressourcen, die Emissionen und die damit verbundenen Risiken aus.

Die Umsetzung ökologischer Zielstellungen in den logistischen Funktionsbereichen hat nachweisbare Kostenreduzierungen zur Folge. Um diese zu erreichen, muss das bislang ökonomisch definierte Zielsystem die ökologischen Ziele der einzelnen Subsysteme integrieren. (Hopfenbeck 1992, S. 962)

Das nachfolgend dargestellte Gesamtzielsystem der umweltorientierten innerbetrieblichen Logistik ergibt sich aus der Gegenüberstellung des ökonomischen Zielsystems der innerbetrieblichen Logistik und dem des Umweltmanagements 


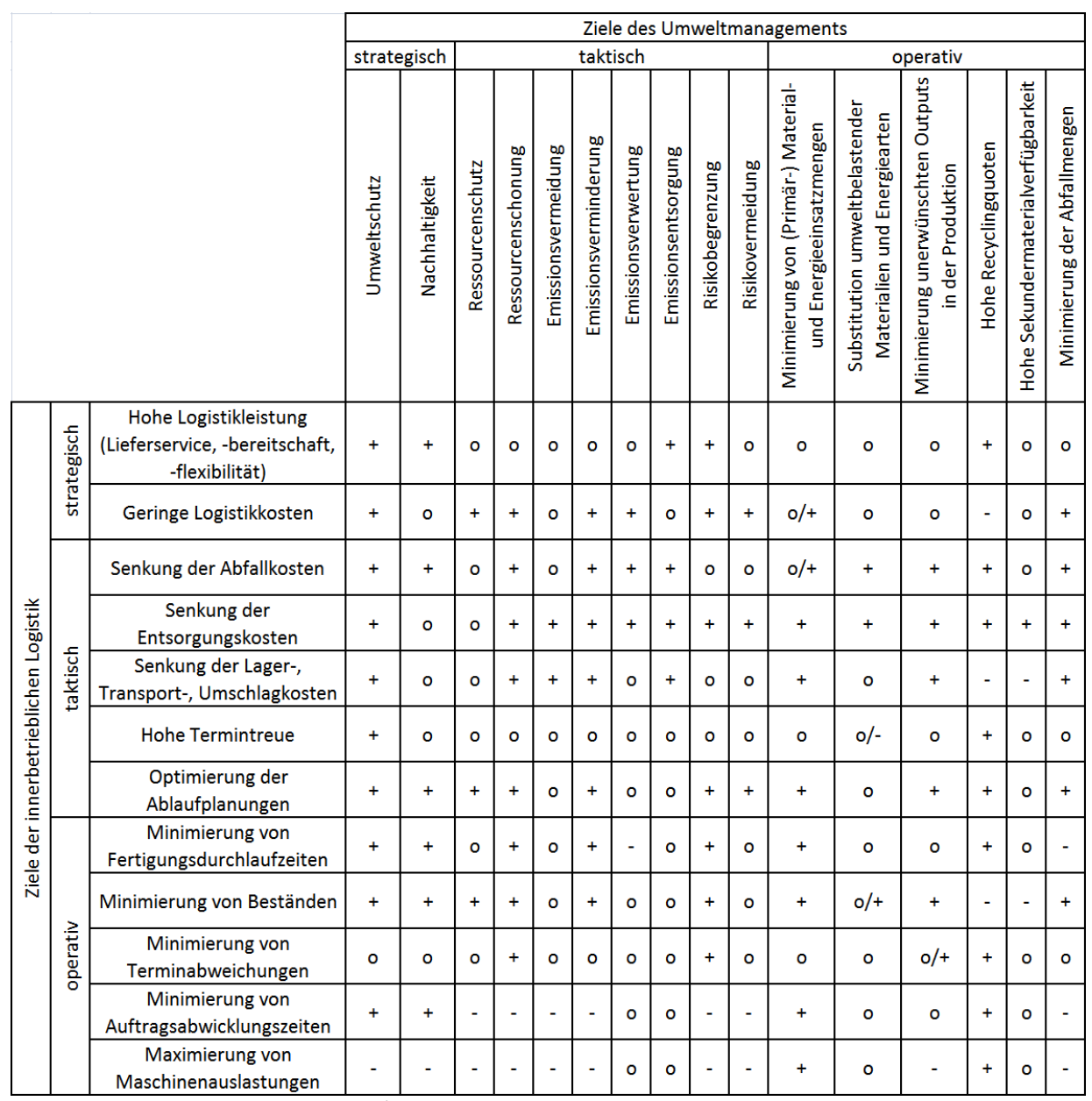

Abbildung 3: Ganzheitliches Zielsystem einer umweltorientierten innerbetrieblichen Logistik

Der hohe Abstraktionsgrad des Gesamtzielsystems macht zwar die Auskunft, ob die genannten Einzelziele in komplementären, neutralen oder konfliktären Beziehungen zueinander stehen, schwierig, dennoch ist es erforderlich, jede einzelne Zielbeziehung zu bewerten und die sich daraus ergebenden Konsequenzen abzuschätzen.

Eine praktische Umsetzung des Zielsystems einer umweltorientierten innerbetrieblichen Logistik in konkrete Strategien und Maßnahmebündel wird vergleichsweise einfach, wenn die Zielbeziehungen einen komplementären Charakter besitzen. In diesem Fall unterstützt die Verfolgung des einen Ziels das Erreichen des anderen. Wenn somit mit der Realisierung ökonomischer Ziele gleichzeitig ökolo- 
gische erreicht werden, sind keine besonderen zusätzlichen Aktivitäten zur Umsetzung der angestrebten ökologischen Ziele einzuleiten.

Neutrale Ziele sind unabhängig voneinander und beeinflussen sich gegenseitig nicht. Sie müssen jeweils getrennt voneinander verfolgt werden.

Bei konfliktären Zielbeziehungen ist zunächst zu untersuchen, ob das ökonomische bzw. das ökologische Einzelziel zugunsten des jeweils anderen aufgegeben werden soll. In der Praxis wird eine solche Entscheidung wohl stets zugunsten des ökonomischen Einzelziels ausfallen, da solche Ziele in den Unternehmensleitungen höhere Priorität besitzen. Sollen Umweltziele auch bei konfliktären Zielbeziehungen Bestand haben, sind beide Einzelziele in der Art zu relativieren, dass jeweils nicht das Optimum der Zielerreichung angestrebt wird. D. h. dass diese Ziele nicht in ihrem höchsten Ausmaß, sondern nur in einem angemessenen Umfang verfolgt werden.

Ein erster Überblick über das Gesamtzielsystem zeigt auf, dass die Anzahl der komplementären und neutralen Zielbeziehungen die der konfliktären deutlich übertrifft - nur ca. 12\% der Zielbeziehungen sind konfliktär. Daraus lässt sich die Annahme ableiten, dass das Gesamtzielsystem der umweltorientierten innerbetrieblichen Logistik grundsätzlich realisierbar ist.

Für die ca. $42 \%$ der Zielbeziehungen mit komplementärem Charakter sind keine besonderen Anstrengungen zur Durchsetzung ökologischer Ziele erforderlich. Allerdings machen die ca. 46\% der neutralen Zielbeziehungen zusätzliche Umsetzungsmaßnahmen erforderlich.

Weiterhin ist zu erkennen, dass weder auf der strategischen noch auf der taktischen Managementebene Zielkonflikte bestehen. Aufgrund der Globalität der Oberziele sind deren Zielbeziehungen selten konfliktär. Auch bei den Zwischenzielen der taktischen Ebene liegen keine Zielkonflikte zwischen den ökonomischen und ökologischen Einzelzielen vor. Bei dem Streben, ökonomische Ziele zu erreichen, werden ökologische unterstützt, mindestens aber nicht negativ beeinflusst. Beispielsweise werden durch Maßnahmen zur Senkung der Entsorgungskosten auch Ziele wie Ressourcenschonung, Emissionsvermeidung und -verminderung unterstützt. Andererseits beeinflussen oder behindern Aktivitäten zur Umsetzung einer hohen Termintreue ökologisch orientierte Ziele wie Ressourcenschutz, schonung etc. nicht

Um dem Umweltschutzgedanken auch bei konfliktären Zielbeziehungen Rechnung zu tragen, können Unterziele beispielsweise in der Weise relativiert werden, dass angemessen hohe Recyclingquoten bei weitgehenden Bestandsreduzierungen anzustreben sind. Weiterhin können Minimierungen von Lagerbestandsmengen und damit Kostenreduzierungen durch Typenbeschränkungen und Normierungen von Materialien erreicht werden. Das führt auch zu ökologischen Entlastungen in den Unternehmen. Eine Reduzierung der Materialvielfalt kann somit zu ökologisch optimierten Lagern beitragen. 


\section{Schlussfolgerungen}

Mit der innerbetrieblichen Logistik wurde ein wichtiger Funktionsbereich von Unternehmen detailliert untersucht. Es konnte gezeigt werden, dass für diesen Bereich ein ökonomisch-ökologisch orientiertes Zielsystem realisierbar ist. Obwohl dieses Ergebnis zur Annahme berechtigt, dass solche Zielsysteme auch für die anderen betrieblichen Funktionsbereiche entwickelt werden können, bedarf diese These jedoch weiterer konkreter Untersuchungen.

Es ist gelungen, das Leitbild der Nachhaltigkeit - soweit deren ökonomische und ökologische Dimensionen angesprochen sind - bezogen auf einen wichtigen betrieblichen Funktionsbereich operationabel zu machen. Damit ist nachgewiesen, dass die nach wie vor in der Praxis vom strategischen Management der Unternehmen vernommene Überzeugung, dass betriebliche Umweltschutzaktivitäten zu kostenintensiv und zu unwirtschaftlich seien, offensichtlich unrichtig ist. Für die gleichzeitige Verfolgung ökonomischer und ökologischer Zielvorstellungen lassen sich positive Synergieeffekte nachweisen.

Für eine effiziente Umsetzung von Zielvorstellungen und Zielsystemen werden häufig Informationssysteme entwickelt und eingesetzt. Grundsätzlich decken die aus der Wirtschaftsinformatik bekannten betrieblichen Informationssysteme (BIS) und BUIS identische betriebliche Funktionsbereich ab, also die Beschaffung, Produktion und den Vertrieb. Dadurch kann der Eindruck einer inhaltlichen Doppelung von Informationssystemen entstehen. Allerdings führt die ökonomische Dimension der nachhaltigen Entwicklung zu einer wert- oder kostenorientierten Sichtweise auf die jeweiligen Funktionsbereiche, während die ökologische Dimension zunächst mengenorientiert ist. Erst mittelbar führt die Realisierung von Zielen wie Material- und Energieeffizienz, sowie Abfall- und Emissionsminimierung zur ökonomischen Dimension der Kostenreduzierung. Die divergierenden Sichtweisen der beiden Dimensionen führen zu unterschiedlichen Ausprägungen der jeweiligen Informationssysteme und damit zur Berechtigung der Existenz zweier unterschiedlich ausgeprägter Informationssysteme für die jeweiligen betrieblichen Funktionsbereiche.

Im Lichte der Ergebnisse der Nachhaltigkeitsdebatte zeigt sich, dass die traditionellen Definitionen von BUIS obsolet geworden sind - sie sind nicht länger „Stand der Kunst“. Die überkommenen Definitionen unterstützen die Umsetzung der operativen Zielvorstellungen und - damit verbunden - die Verfolgung isolierter, nicht aufeinander abgestimmter Einzelziele. Wie viele der in der Praxis eingesetzten Anwendungssysteme demonstrieren, führt diese Sichtweise zur Realisierung sog. Insellösungen, somit zu Softwaresystemen, die ohne jegliche Integrationseffekte unverbunden nebeneinander existieren.

Der mit dem Informationsmanagement verbundene ganzheitliche Zugang zur Gestaltung und Realisierung von Informationssystemen ist somit auch innerhalb der Betrieblichen Umweltinformatik zwingend erforderlich, weil es offenbar nur so gelingen kann, die bisherige operative Ausrichtung der BUIS zu überwinden. 


\section{Literatur}

Balzert H (1996) Lehrbuch der Software-Technik: Software-Entwicklung. Heidelberg et al.

Beucker Severin et al.(2002) Betriebliche Umweltinformationssysteme und ihre Funktion für die Ressourceneffizienz, Ergebnisbericht 2.3 des Projekts CARE - Computergestützte Ressourceneffizienzrechnung in der mittelständischen Wirtschaft. Stuttgart

Bundesministerium für Umwelt, Naturschutz und Reaktorsicherheit (2001) Umweltbundesamt: Handbuch Umweltcontrolling. München.

Deutscher Bundestag (Hrsg.) (1997): Konzept Nachhaltigkeit. Fundamente für die Gesellschaft von morgen; Zwischenbericht der Enquetekommission „Schutz des Menschen und der Umwelt - Ziele und Rahmenbedingungen einer nachhaltig zukunftsverträglichen Entwicklung“" des 13. Deutschen Bundestages. Bonn

El-Gayar O, Fritz BD (2006) Environmental Management Information Systems for Sustainable Development: A Conceptual Overview, in Communication of the AIS 17.

Eschenbach R, Neumann K (1995) Betriebliche Umweltinformationssysteme. Mainz, Wien

Goetz F, Czymmek F (2002) Der Beitrag des Öko-Rating für die unternehmerischen Stakeholder. In: Beuermann G (Hrsg.) 12. Arbeitsbericht zum Umweltmanagement. Köln.

Haasis HD (2001) Unternehmensführung und nachhaltiges Wirtschaften. In: von Fischer, Hajo; Schwiering, Dieter (Hrsg.) Unternehmensführung im Spannungsfeld zwischen Finanz- und Kulturtechnik: Handlungsspielräume und Gestaltungszwänge. Hamburg.

Hamschmidt J (2001) Wirksamkeit von Umweltmanagementsystemen - Stand der Praxis und Entwicklungsperspektiven. Diss. St. Gallen.

Hauff V (Hrsg.) (1987) Unsere gemeinsame Zukunft, Bericht der Weltkommission für Umwelt und Entwicklung, deutsche Fassung. Greven

Heiserich O-E (2002) Logistik. Eine praktische Einführung, 3. Auflage. Wiesbaden

Heß HJ (2007) Nachhaltigkeitsmanagement und nachhaltige Business Cases bei Finanzkonzernen. Das Management ökologischer und sozialer Probleme durch die Unternehmenszentralen. Dis., Berlin

Hilty L (1997) Umweltbezogene Informationsverarbeitung - Beiträge der Informatik zu einer nachhaltigen Entwicklung. Habil., Hamburg 
Hilty L, Rautenstrauch C (1995) Betriebliche Umweltinformatik. In: Page, Hilty (Hrsg.): Betriebliche Umweltinformatik. München.

Hopfenbeck W (1992) Allgemeine Betriebswirtschafts- und Managementlehre. Das Untenehmen im Spannungsfeld zwischen ökonomischen, sozialen und ökologischen Interessen, 6. Auflage. Landsberg/Lech

Junker H (Hrsg.) (2004) Werkstattberichte aus der Betrieblichen Umweltinformatik, Band 1. Aachen.

Junker H, Lang CV (Hrsg.) (2006) Werkstattberichte aus der Betrieblichen Umweltinformatik, Band 3. Aachen.

Junker H, Molloy I (Hrsg.) (2007) Werkstattberichte aus der Betrieblichen Umweltinformatik, Band 4. Aachen.

Junker H, Rey U (Hrsg.) (2005) Werkstattberichte aus der Betrieblichen Umweltinformatik, Band 2. Aachen.

Kramer J (1993) Betriebliche Umweltinformationssyteme (BUIS): Voraussetzung effektiven Umweltmanagements. In: Arndt HK (Hrsg.)

Umweltinformationssysteme für Unternehmen, Schriftenreihe des IÖW 69/93 Berlin

Lang C (2007a) Ökonomisch-ökologische Zielsysteme als Herausforderung für das Ressourcenmanagement. In: Pietsch T, Lang C (Hrsg.)

Ressourcenmanagement. Berlin.

Lang C (2007b) Konzeption eines Referenzmodells für betriebliche Umweltinformationssysteme im Bereich der innerbetrieblichen Logistik. Aachen.

Müller-Christ G (2001) Umweltmanagement. Umweltschutz und nachhaltige Entwicklung. München.

Rautenstrauch C (1997) Fachkonzept für ein integriertes ProduktionsRecyclingplanungs- und Steuerungssystem (PRPS-System). Berlin.

Rautenstrauch C (1999) Betriebliche Umweltinformationssysteme: Grundlagen, Konzepte und Systeme. Berlin.

Rey U, Schnapernelle D (1999) Transformationsplattform zur Darstellung ITgestützter Werkzeuge im betrieblichen Umweltschutz. In: Dade C, Schulz B (Hrsg.) Management von Umweltinformationen in vernetzten Umgebungen. Marburg

Rösch A, Grossmann WD (1998) Nachhaltigkeit in der Informationsgesellschaft. In: Zeitschrift für angewandte Geographie, Vol. 22, Nr. 3. 
Schlatter A (2000) Der ökonomische Nutzen Betrieblicher Informationssysteme Eine Evaluationsmethode. In: Hilty L, Schulthess D, Ruddy TF (Hrsg.)

Strategische und betriebsübergreifende Anwendungen betrieblicher Umweltinformationssysteme, „Umweltinformatik aktuell“, Bd. 25. Marburg

Schulz W (1991) Betriebliche Umweltinformationssysteme für eine zukunftsweisende Unternehmensplanung. In: Grünwald W (Hrsg.) AGPLANHandbuch zur Unternehmensplanung Band 4 Berlin

Teubner RA (1999) Organisations- und Informationssytemgestaltung. Theoretische Grundlagen und integrierte Methoden. Münster Diss.

Teuteberg F, Straßenburg J (2009) State of the Art and Future Research in Environmental Management Information Systems - A Systematic Literature Review. In: Athanasiadis IN, Mitkas PA, Rizzoli AE, Marx Gomez J (Hrsg.): Information Technologies in Environmental Engineering, Proceedings of the 4th International ICSC Symposium, Thessaloniki.

Wöhe G (2005) Einführung in die Allgemeine Betriebswirtschaftlehre, 22. Auflage. München

Zahn E, Steimle V (1993) Umweltinformationssysteme und umweltbezogene Strategieunterstützungssysteme. In: Wagner GR (Hrsg.) Betriebswirtschaft und Umweltschutz, Stuttgart 